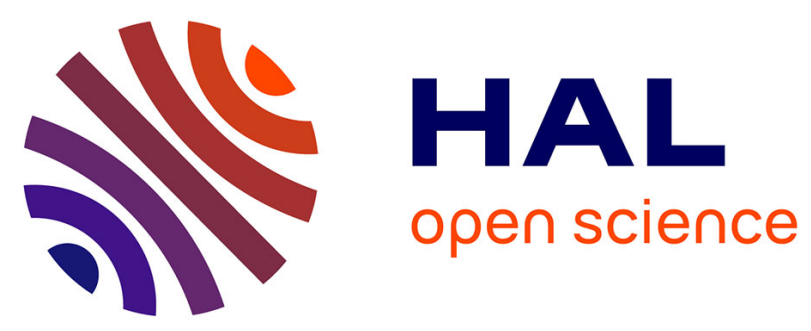

\title{
An automatic cell culture platform for differentiation of human induced pluripotent stem cells
}

Gabriele Pitingolo, Yong He, Boxin Huang, Li Wang, Jian Shi, Yong Chen

\section{To cite this version:}

Gabriele Pitingolo, Yong He, Boxin Huang, Li Wang, Jian Shi, et al.. An automatic cell culture platform for differentiation of human induced pluripotent stem cells. Microelectronic Engineering, 2020, 231, pp.111371. 10.1016/j.mee.2020.111371 . hal-03013336

\section{HAL Id: hal-03013336 https://hal.science/hal-03013336}

Submitted on 18 Nov 2020

HAL is a multi-disciplinary open access archive for the deposit and dissemination of scientific research documents, whether they are published or not. The documents may come from teaching and research institutions in France or abroad, or from public or private research centers.
L'archive ouverte pluridisciplinaire HAL, est destinée au dépôt et à la diffusion de documents scientifiques de niveau recherche, publiés ou non, émanant des établissements d'enseignement et de recherche français ou étrangers, des laboratoires publics ou privés. 


\title{
An automatic cell culture platform for differentiation of human induced pluripotent stem cells
}

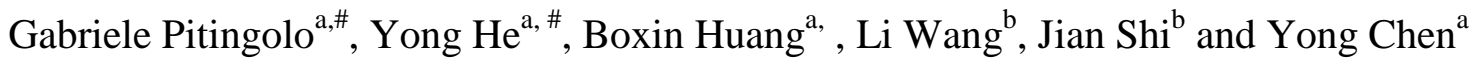 \\ ${ }^{a}$ PASTEUR, Département de chimie, École normale supérieure, PSL University, \\ Sorbonne Université, CNRS, 75005 Paris, France \\ ${ }^{\mathrm{b}}$ MesoBioTech, 231 Rue Saint-Honoré, 75001 Paris, France \\ *E-mail: yong.chen@ens.fr \\ ${ }^{\#}$ These authors contributed equally
}

\begin{abstract}
We developed an automatic cell culture platform to facilitate the research and development of stem cell differentiation. This platform consists of a distributor of culture media containing different cell factors, a culture unit allowing either static or dynamic exchange of the medium and a central unit for pumping and process control, respectively. This platform was firstly used for cell culture with a tissue culture dish at a high medium exchange rate, showing an increased cell growth due to enhanced uptake of the nutrients and enhanced clearance of the metabolites. Then, the platform was used for automatic differentiation of human induced pluripotent stem cells (hiPSCs) toward cardiomyocytes which involved six types of culture media. As results, functional cardiomyocytes could be obtained after automatic processing during two weeks, showing regular beating over a large area. Details are described to show the applicability of the platform to other types of stem cell differentiation or cell-based assays.
\end{abstract}

Keywords: Automation, cell culture, Stem cell differentiation, Tissue engineering, 


\section{Introduction}

Cell proliferation and stem cell differentiation are essential for many cell-based assays and organ on-a-chip applications [1,2]. In particular, differentiation of human induced pluripotent stem cell (hiPSC) can give rise to different types of cells that can be used for regenerative medicine, drug discovery or cell therapies [3-8]. Despite their high potential, it is still challenging to control the quality of hiPSCs and their derivatives. One of the possible reasons is that the proposed protocols have been mostly established by using conventional tools and techniques. Differentiation of hiPSCs requires sequential operations over a long period for culture medium preparation and exchange, substrate replacement and cell passages that are often laborious and cumbersome [9]. Automatic differentiation of hiPSCs has been demonstrated using commercial bioreactors for different target cell types, including cardiomyocytes [10], macrophages [11], alveolar epithelium [12], and neurons [13]. However, most bioreactors are limited to suspension culture, in which cells are in an agitated growth medium with or without micro-carriers and cells are subjected to constant perturbations, including shear stress and gravitational force changes. Large platforms for automatic hiPSC self-renewing [14] and differentiation [15,16] have also been reported, which were essentially based on large-scale machinery for robotic manipulation of culture wares. These platforms are generally expensive and difficult to change the culture conditions. For example, the system proposed by Sartorius allows standard operations with large batches for which the medium change relies on a serious processes, including retrieving $\mathrm{T}$-flask from the incubator, removing $\mathrm{T}$ flask cap, pouring media into a waste funnel, adding new medium, and replacing T-flask in the flask carousel incubator, with the help of robot, shaking machine and pumps [16]. This system can be used in highly specially research centers or industries but not suited for common laboratory uses. As in the current stage of hiPSCs development, there are still many challenges requiring flexible and quick access on process automation, a laboratory platform is desirable.

In this work, we developed a laboratory platform for automated cell proliferation and hiPSC differentiation. This platform consists of a storage unit for selected medium distribution from a refrigerator, a culture unit for static or dynamic culture medium exchange in an incubator, and a control unit for programing and control of the whole process. We show that this platform is compatible with culture dish and culture patch devices. When it was used for dynamic culture where the culture medium was exchanged at a high frequency, the culture was performed under a perfusion-diffusion regime. When it was used for differentiation of human induced pluripotent stem cells, cardiomyocytes with regular beating, for example, could be obtained. The laboratory platform we developed is compact and cost-effective. It does allow not only reducing the manpower and 
reagent consumption but also minimizing risks of operation error and contamination. It can operate for fast and accurate processing of soluble cell factors, thereby enlarging the windows for protocol design and processing parameter optimization. More generally, this platform would allow investigating the effects of culture substrates and the influence of culture flow conditions. It should also be applicable to organ-on-a-chip systems.

\section{Experimental}

\subsection{Design and development of the automated cell culture platform}

As shown in Fig. 1, the culture platform is composed of three parts: i) Storage unit, ii) Control unit, and iii) Cell culture unit. The storage unit was designed to keep the culture media at low temperature in standard falcon tubes (M1-M6) and to distribute them upon request. The cap of each tube has an inlet connected to rotating valve $\mathrm{Ra}$ and an outlet connected to rotating valve $\mathrm{Rm}$. The cell culture unit consists of two peristaltic pumps $(3,4)$, two falcon tubes $(\mathrm{R}, \mathrm{W})$, and a culture dish plate where a metallic handling plate is fixed above the dish cover. The control unit is also equipped with two peristaltic pumps $(1,2)$, but more importantly an ATmega 2560 based microcontroller (Arduino) and associated electronic components (keyboard, OLED display, etc.). The four pumps (1-4) and two valves ( $\mathrm{Ra}, \mathrm{Rm}$ ) are all controlled by the microcontroller with RS485 communication and silicone tubing with standard Luer connectors are used for as pipelines of the culture media.

During cell cultivation, the storage unit is kept at $4{ }^{\circ} \mathrm{C}$ in a refrigerator and the cell culture unit is placed in an incubator at $37^{\circ} \mathrm{C}$ with $5 \%$ humidified $\mathrm{CO}_{2}$. When a culture medium is selected, the two rotating valves are simultaneously switched to the positions where filtered air can come in the tube and the medium can be pumped out. The medium is firstly injected into tube $\mathrm{R}$ by pump 1 . When tube $\mathrm{R}$ is filled, the medium is injected into the culture dish by pump 3 for incubation. Afterward, the medium is transported into tube $\mathrm{W}$ by pump 4.

Fig. 2 shows photographs of the three units of the laboratory platform with fluidic pipelines (a), a dish cover handling plate (b), a culture patch (c), and a patch mounting device (d). Here, two female Luer locks to panel mount with barb are fixed on the cover handling plate for injection and aspiration of the culture medium (b). The culture patch is made of monolayer of gelatin nanofibers deposited on a honeycomb micro-frame (see below). The patch mounting device in polytetrafluoroethylene (PTFE) is used to localize 6 patches and place them together in the culture dish. 
Cell culture and stem cell differentiation sequences are programmed in Arduino $\mathrm{C}$ with a PC and then transferred to the memory of Arduino board through a USB cable. The processing parameters and sequences are shown on the OLED display of the control unit, which can be modified or chosen with the keyboard. During automatic processes, a sequence can be stopped or paused for manual intervention. In addition, the control unit integrated a Wireless or Bluetooth module for eventual remote control.

\subsection{Fabrication of the culture patch}

The same protocol of our previous work has been employed for the fabrication of the culture patch for the proposed automated platform [17]. Briefly, the patch consists of a monolayer of crosslinked gelatin nanofibers supported by a polyethylene glycol diacrylate (PEGDA Mw=250, Sigma) honeycomb frame (Fig. 2b). The PEGDA frame was fabricated with a UV based molding method, the gelatin nanofibers were obtained by electrospinning. A pattern of honeycomb frame was produced on a chromium mask with a micropattern generator ( $\mu \mathrm{PG}$ 101, Heidelberg Instruments, Germany). A photoresist layer of $50 \mu \mathrm{m}$ thickness (SU 8, MicroChem, France) was then spin-coated on a silicon wafer with the chromium mask and exposed with UV light. After development, for anti-sticking treatment the mask was placed in trimethylchlorosilane (TMCS) vapor for $1 \mathrm{~min}$. A Polydimethylsiloxane (PDMS) solution composed of a pre-polymer and crosslinker (GE RTV 615, GE, France) at a weight ratio of 10:1 was prepared and poured onto the photoresist mold. After curing $\left(75^{\circ} \mathrm{C}\right.$ for $\left.2 \mathrm{~h}\right)$, the PDMS layer was peeled off and placed on a glass slide. After degassing of the mold for $15 \mathrm{~min}$, PEGDA with 1 v/v\% photo-initiator (Irgacure 2959, Sigma) was introduced to fill the PDMS-glass cavity, followed by $2 \mathrm{~min}$ UV exposure (23 $\mathrm{mW} / \mathrm{cm}^{2}$ ). Finally, the solidified PEGDA honeycomb was separated from the PDMS mold and gelatin nanofibers were electrospun on the PEGDA frame.

\subsection{Finite-element modeling}

The software COMSOL Multiphysics was used to simulate the gradient concentration in a tissue culture dish of $60 \mathrm{~mm}$ diameter under both static and dynamic culture conditions. Firstly, we established the fluid domain by SolidWorks and imported it to COMSOL Multiphysics. Then, we chose simulation modules "Creeping flow" and "Transport of Diluted Species" for numerical simulation based on Finite-Element Method (FEM). The simulation results are reported as a pattern of solute concentration in the culture dish after reaching a steady state. 


\subsection{Preparation of the platform}

Before cell cultivation, tubing and connectors were sterilized (falcon tubes are commercial and sterile products). All fluidic connections and cell seeding were performed in a laminar air flow hood. After assembling of the three units of the platform, a washing sequence was launched to sterilize all pipelines and liquid passages (silicon tubing, rotating valves, and dish cover handling plate) with a $70 \%$ ethanol solution. This washing cycle was repeated two times and the ethanol solution was recovered in falcon tube $\mathrm{W}$. Thereafter, the same sequence was applied with pen-strep solution (1\%) and culture medium to stabilize the system before cell cultivation. A flow rate of 3 $\mathrm{mL} / \mathrm{min}$ was set for all washing cycles.

\subsection{Cell culture test}

HeLa S3 cells were used for culture test with DMEM medium supplemented with $10 \%$ fetal bovine serum (FBS), $1 \%$ Penicillin-Streptomycin (pen-strep) and $1 \%$ L-Glutamine. They were seeded on a $60 \mathrm{~mm}$ culture dish with a cell number of $2 \times 10^{5}$. Then, the dish was placed in the cell culture unit and cells were incubated in a $5 \%$ humidified $\mathrm{CO}_{2}$ incubator at $37^{\circ} \mathrm{C}$. After static incubation for $2 \mathrm{~h}$, an automatic culture sequence was launched. Pump 1 and valves Ra and Rm were used to fill the falcon tube R every two days, while pumps 3 and 4 were used to circulate the culture medium inside the incubator: the medium of falcon tube $\mathrm{R}$ was firstly pumped out and injected into the dish by pump 3, it was then pumped from the dish to the falcon tube W by pump 4 . Since pump 3 and 4 could operate simultaneously, a perfusion culture regime could be created when the medium pumped from the dish was injected into the falcon tube R instead of W. In such a case, a perfusion-diffusion culture regime could also be achieved by applying a run-rest sequence with a small duty cycle. Here, a sequence of $4 \mathrm{~s}$ run -1 min rest was tested. A control experiment was performed by manually exchanging of the same amount of culture medium every 2 days. Cells growth was monitored, using inverted light microscope, for several days. Pictures were taken after 1, 24, 48, 72 and 96 hours. Quantitative analysis of proliferation rate and confluence were performed using free software ImageJ (NIH USA). Here, a threshold function was used to calculate the cell covered area at day 1,2 and 3 and a confluence state was obtained once he whole dish area was full covered.

\subsection{Cardiac differentiation}

HiPSCs of cell line 253G1 were cultured with complete E8 medium (M1) in a culture dish coated with 1:100 diluted vitronectin (Thermo Fisher, France) at $37{ }^{\circ} \mathrm{C}$ in $5 \% \mathrm{CO}_{2}$. The medium 
was renewed every day until the cells reached about $80 \%$ confluence. Cells were then collected by dissociation using a $0.5 \mathrm{mM}$ EDTA solution. To promote the adhesion of hiPSCs on gelatin fibers, the culture patch was coated with vitronectin diluted in DPBS at a ratio of 1:500 and then placed in a culture dish. $2 \times 10^{5}$ hiPSCs in $50 \mu \mathrm{E} 8$ medium containing $10 \mu \mathrm{M}$ Y-27632 (M2) (ROCK inhibitor, Hello Bio, UK) were dropped on the culture patch and then incubated for $1 \mathrm{~h}$. Thereafter, the culture dish was connected to the circuit of the automated culture platform and the mediums and factors were changed automatically. Briefly, the M2 medium was automatically replaced by RPMI 1640 medium containing B27 minus insulin supplement and $12 \mu \mathrm{M}$ of CHIR99021 (STEMCELL Technology) (M3). After $24 \mathrm{~h}$ incubation, the M3 was replaced with RPMI 1640/B27 without insulin (M4). After another $48 \mathrm{~h}$, the M4 was replaced by RPMI 1640/B27 without insulin but containing $5 \mu \mathrm{M}$ IWP2 (Hello Bio) (M5). After $48 \mathrm{~h}$ in incubator, the M5 was replaced with M4. After 48 h, the M4 was replaced by RPMI 1640/B27 (M6) and changed every three days. Following the same protocol, hiPSCs cultured on patches with 1:100 diluted vitronectin coating were differentiated into cardiomyocytes, changing the mediums and factors manually, as control. The video microscope was used for video capturing. After recovering the culture dish from the automated cell culture platform, video recordings of the cardiomyocytes beating were captured for 10 and 60 seconds. All recordings were carried out in the cell culture laboratory.

\section{Results and discussion}

The culture platform developed in this work is compact, modular, versatile, and useful for large variety of experiments. The storage unit can be placed in a traditional refrigerator and the culture unit can be placed in a conventional incubator. For long period operation, it is possible to place only the culture media in a refrigerator of lower temperature whereas the rest of the unit can be put outside the refrigerator. The flow rate of peristaltic pumps can vary over a large range from 0.01 to $16 \mathrm{~mL} / \mathrm{min}$.

Numerical simulation has been performed with the fluid domain as shown in Fig 3a and a solute of a diffusion coefficient of $6 \times 10^{-10} \mathrm{~m}^{2} / \mathrm{s}$. Under static culture conditions (diffusion dominant), cells grow on the surface of the dish and the solute concentration should be highest on the top level of the medium surface and lowest on the cell layer. With a homogenous solute concentration of $25 \mathrm{~mol} / \mathrm{m}^{3}$ and a medium height of $2 \mathrm{~mm}$. A steady stage could be reached in 1 min. As can be seen in Fig. 3b by the color ribbons, the concentration varies from bottom to upper surface in a range of $12 \sim 25 \mathrm{~mol} / \mathrm{m}^{3}$, which means that in the proximity of the cell layer the concentration of nutrients is only half of that of the top surface. On the contrary, the concentration of cellular metabolites in the 
proximity of the cell layer is larger than that of the top surface. Under dynamic culture conditions (perfusion dominant), we assumed an initial concentration of $25 \mathrm{~mol} / \mathrm{m}^{3}$ at the inlet and 0 solute at the outlet. After one minute with a flow rate of $20 \mu \mathrm{l} / \mathrm{s}$ for both inlet and outlet, a steady state could be reached. As shown in Fig. $3 b$ by the color ribbons, the simulation suggests a quite uniform solute concentration over the whole medium layer, due to continuous renewal of the medium in the dish. Similarly, the concentration of the metabolites should also be homogenous. Thus, we can conclude that dynamic culture may enhance both nutrient uptake and metabolite clearance of the cells which gives rise to an increased cell proliferation rate. More generally, a perfusion-diffusion regime can be reached by alternating static and dynamic culture conditions.

Figure 4a shows microphotographs of Hela cells after incubation for 1, 24, 48, 72 and 96 hours in both cases of automatic and manual culture, respectively. Here, automatic culture means not only automatically renew the culture medium in falcon tube $\mathrm{R}$ every two days but also perfuse the culture medium in the dish quasi-continuously over the whole period of culture. Clearly, the HeLa cells cultivated under perfusion-diffusion regime grew more rapidly than static culture and they reached confluent after $72 \mathrm{~h}$, in contrast that after $96 \mathrm{~h}$ with manual renewal of the culture medium. Figure $4 \mathrm{~b}$ shows a comparison between the two types of culture after incubation for $96 \mathrm{~h}$, the empty spaces indicated by white arrows suggested a lower confluence quantity of static culture. The microphotographs of both automatic and manual culture were further analyzed by ImageJ, showing remarkable differences in proliferation (Fig. 4c). This is due probably to the enhanced uptake of nutrients and enhanced clearance of the metabolites, thanks to the quasi-continuous renewing of the culture medium.

The automated differentiation of hiPSCs toward functional cardiomyocytes has been tested with several culture patches using PTFE patch mounting device. Once patches were fixed, the device was placed in a conventional $60 \mathrm{~mm}$ culture dish. This mounting device is particularly useful which guarantees a uniform iPSCs culture and optimum cardiac differentiation. To illustrate the high potential of the system, we adopted a differentiated protocol previously developed for cardiac differentiation [18]. The process flow is in Fig. 5, which consists of three parts: i) system preparation, ii) hiPSCs culture and embryoids (EBs) formation and iii) cardiac differentiation. Particular attention has to be paid to the system preparation, since this part involves both manual and machine-controlled operation and any contamination has to be avoided.

By using our laboratory platform with a hiPSC differentiation protocol previously defined [19], we could be able to produce automatically functional cardiomyocytes on a patch (Fig. 6). During the early stage of the process, cells migrated progressively away from the hemisphere 
colonies to form a cell sheet at day 4 and then showed up started irregular beating around day 9. At day 14, the cardiac contraction became robust and homogeneous over the whole patch area (Movie $\mathrm{S} 1-\mathrm{S} 2 \dagger)$. Here, the contraction profiles were plotted by simply measuring the time course of the image intensity change in a small ROI of the movies (Fig. 6b and 6c). As can be seen in Fig. 6d, the beating rate of automatically processed cells is slightly higher than that by manual operations. Since the same differentiation protocol was used in both cases, this difference in beating frequency may be attributed to the difference in off-ground distance of the patches: it was simply placed in the dish in the case of manual culture (short distance) but mounted in a supporting device in the case of automatic processing (Fig. 2d) (large distance). It is known that the beating rate of cardiomyocytes increases with time during the maturation phase, suggesting that the cardiomyocytes obtained by automatic processing have improved maturation than that of manual one, due probably to the enhanced uptake of nutrient and the enhanced clearance of metabolites.

We would like mention that other parameters such as the elasticity and anisotropy of the culture substrates as well as the flow rate of the medium are also important for cardiac differentiation of hiPSCs, this work was limited to the design of the apparatus itself and the automated protocol. We believe that this laboratory platform is a useful alternative to the more conventional bioreactors and the large automated systems in terms of cost, flexibility and versatility. As demonstrated, this platform is compatible to culture dish and culture patches. Its application can also be extended to organ on-a-chip applications, thereby offering a new perspective for future cell-based assays.

\section{Conclusions}

In this paper, a cost-effective, modular and compact platform for automated cell culture and hiPSC differentiation has been proposed and validated for cell culture and hiPSC differentiation. We demonstrated that under perfusion-diffusion regime Hela cells have a higher growth rate on dish with respect to conventional static culture. We also demonstrated the feasibility of automated differentiation of hiPSCs toward functional cardiomyocytes on patches. We believe that this laboratory platform is a valuable alternative to manual processing and robotic based large-scale culture platforms, enlarging its application capability to a variety of micro-engineered substrates. Further studies and applications are expected in order to fully explore the potential of such a development. 


\section{Acknowledgment}

This work was supported by Agence de Recherche Nationale under contract ANR-17-CE09-0017 (AlveolusMimics), European Commission Cost Action BIONECA (CA 16122), DIM ELICIT program of Ile-de-France, and PSL Valorization through Pre-maturation project. Yong $\mathrm{He}$ is grateful to the China Scholarship Council for grant of his $\mathrm{PhD}$ studies. Boxin Huang is grateful to the Oversea Study Program of Guangzhou Elite Project.

\section{References}

[1] A. Astashkina, B. Mann, D.W. Grainger, A critical evaluation of in vitro cell culture models for highthroughput drug screening and toxicity, Pharmacology \& therapeutics 134(1) (2012) 82-106.

[2] S.A. Langhans, Three-dimensional in vitro cell culture models in drug discovery and drug repositioning, Frontiers in pharmacology 9 (2018) 6.

[3] M. Daniszewski, D.E. Crombie, R. Henderson, H.H. Liang, R.C. Wong, A.W. Hewitt, A. Pebay, Automated cell culture systems and their applications to human pluripotent stem cell studies, SLAS TECHNOLOGY: Translating Life Sciences Innovation 23(4) (2018) 315-325.

[4] J. Yu, M.A. Vodyanik, K. Smuga-Otto, J. Antosiewicz-Bourget, J.L. Frane, S. Tian, J. Nie, G.A. Jonsdottir, V. Ruotti, R. Stewart, Induced pluripotent stem cell lines derived from human somatic cells, Science 318(5858) (2007) 1917-1920.

[5] M. Grskovic, A. Javaherian, B. Strulovici, G.Q. Daley, Induced pluripotent stem cells — opportunities for disease modelling and drug discovery, Nature reviews Drug discovery 10(12) (2011) 915.

[6] L. Zwi-Dantsis, I. Huber, M. Habib, A. Winterstern, A. Gepstein, G. Arbel, L. Gepstein, Derivation and cardiomyocyte differentiation of induced pluripotent stem cells from heart failure patients, European heart journal 34(21) (2012) 1575-1586.

[7] M. Takeda, S. Miyagawa, S. Fukushima, A. Saito, E. Ito, A. Harada, R. Matsuura, H. Iseoka, N. Sougawa, N. Mochizuki-Oda, Development of in vitro drug-induced cardiotoxicity assay by using threedimensional cardiac tissues derived from human induced pluripotent stem cells, Tissue Engineering Part C: Methods 24(1) (2018) 56-67.

[8] A.M. Tukker, F.M. Wijnolts, A. de Groot, R.H. Westerink, Human iPSC-derived neuronal models for in vitro neurotoxicity assessment, Neurotoxicology 67 (2018) 215-225.

[9] E. Ratcliffe, R.J. Thomas, D.J. Williams, Current understanding and challenges in bioprocessing of stem cell-based therapies for regenerative medicine, British medical bulletin 100(1) (2011) 137.

[10] M. Schroeder, S. Niebruegge, A. Werner, E. Willbold, M. Burg, M. Ruediger, L.J. Field, J. Lehmann, R. Zweigerdt, Differentiation and lineage selection of mouse embryonic stem cells in a stirred bench scale bioreactor with automated process control, Biotechnology and bioengineering 92(7) (2005) 920-933. 
[11] M. Ackermann, H. Kempf, M. Hetzel, C. Hesse, A.R. Hashtchin, K. Brinkert, J.W. Schott, K. Haake, M.P. Kühnel, S. Glage, Bioreactor-based mass production of human iPSC-derived macrophages enables immunotherapies against bacterial airway infections, Nature communications 9(1) (2018) 5088.

[12] M. Ghaedi, J.J. Mendez, P.F. Bove, A. Sivarapatna, M.S.B. Raredon, L.E. Niklason, Alveolar epithelial differentiation of human induced pluripotent stem cells in a rotating bioreactor, Biomaterials 35(2) (2014) 699-710.

[13] M. Serra, C. Brito, E.M. Costa, M.F. Sousa, P.M. Alves, Integrating human stem cell expansion and neuronal differentiation in bioreactors, BMC biotechnology 9(1) (2009) 82.

[14] S. Konagaya, T. Ando, T. Yamauchi, H. Suemori, H. Iwata, Long-term maintenance of human induced pluripotent stem cells by automated cell culture system, Scientific reports 5 (2015) 16647.

[15] K.I. Kane, E.L. Moreno, S. Hachi, M. Walter, J. Jarazo, M.A. Oliveira, T. Hankemeier, P. Vulto, J.C. Schwamborn, M. Thoma, Automated microfluidic cell culture of stem cell derived dopaminergic neurons, Scientific reports 9(1) (2019) 1796.

[16] F. Regent, L. Morizur, L. Lesueur, W. Habeler, A. Plancheron, K. Ben M'Barek \& C. Monville, Automation of human pluripotent stem cell differentiation toward retinal pigment epithelial cells for largescale productions, Scientific Reports 9 (2019) 10646

[17] Y. Tang, L. Liu, J. Li, L. Yu, F.P.U. Severino, L. Wang, J. Shi, X. Tu, V. Torre, Y. Chen, Effective motor neuron differentiation of hiPSCs on a patch made of crosslinked monolayer gelatin nanofibers, Journal of Materials Chemistry B 4(19) (2016) 3305-3312.

[18] Y. Tang, L. Liu, J. Li, L. Yu, L. Wang, J. Shi, Y. Chen, Induction and differentiation of human induced pluripotent stem cells into functional cardiomyocytes on a compartmented monolayer of gelatin nanofibers, Nanoscale 8(30) (2016) 14530-14540.

[19] X. Lian, J. Zhang, S.M. Azarin, K. Zhu, L.B. Hazeltine, X. Bao, C. Hsiao, T.J. Kamp, S.P. Palecek, Directed cardiomyocyte differentiation from human pluripotent stem cells by modulating Wnt/ $\beta$-catenin signaling under fully defined conditions, Nature protocols 8(1) (2013) 162. 


\section{Figure captions}

Figure 1. Schematic presentation of the laboratory platform designed for automated cell culture and differentiation of stem cells. Here, the storage unit is placed in a refrigerator (left), the cell culture unit is placed in an incubator (right), and the control unit is used to pump the media from the storage unit to the cell culture unit and to control fluidic motion of all pipelines (middle).

Figure 2. (a) Photographs of the platform, showing a media-distribution unit (left), a control unit (center) and a culture unit (right) with fluidic pipelines according to Fig. 1. (b) Photograph of a metallic holder with inlet and outlet mounted on a normal dish cover. (c) Photograph of a culture patch made of monolayer nanofibers and a ring for handling. (d) Schematic diagram of mounting device for six patches.

Figure 3. Numerical simulation based on COMSOL-Multiphysics: (a) Fluid domain with a culture dish of $60 \mathrm{~mm}$ diameter and a pair of well-separated inlet and outlet; (b) Solute concentration in the dish under static culture condition; (c) Solute concentration in the dish under dynamic culture condition. Simulation parameters are given in section 2.

Figure 4. Phase contrast images (10 x objectives) of HeLa cells under automatic (left column) and manual (right column) culture conditions (a). The percentages of the cell layer confluence under the two types of culture conditions are compared $(b, c)$, showing significant differences due to different growth rates. Scale bars: $50 \mu \mathrm{m}$.

Figure 5. Automated media change pipeline for cardiac differentiation and preparation steps. M1: E8+10 $\mu$ MY27632, M2 = E8, M3: RPMI 1640 + B27 without insulin + $12 \mu \mathrm{m}$ CHIR 99021, M4:

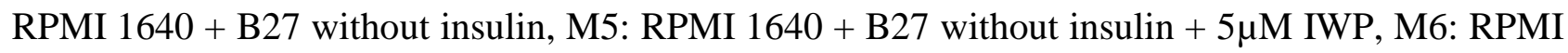
$1640+\mathrm{B} 27$.

Figure 6. On-patch cardiac differentiation of hiPSCs under different culture conditions. (a) Differentiation protocol; (b) Phase contrast images of differentiating hiPSCs after incubation for $2 \mathrm{~h}$ and 1, 4, and 20 days. Cardiac beating profiles of differentiating hiPSCs at day 14 under automatic (c) and manual (d) culture conditions. (e) Variation of the beating rate as function of operation time. 
Fig. 1

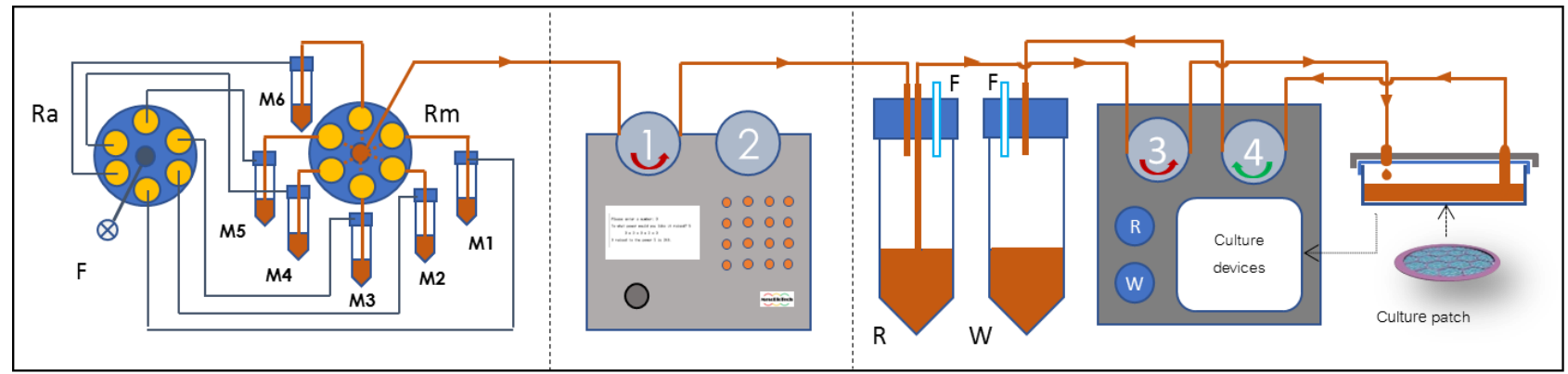

Storage unit

Control unit

Cell culture unit

Fig.2

(a)

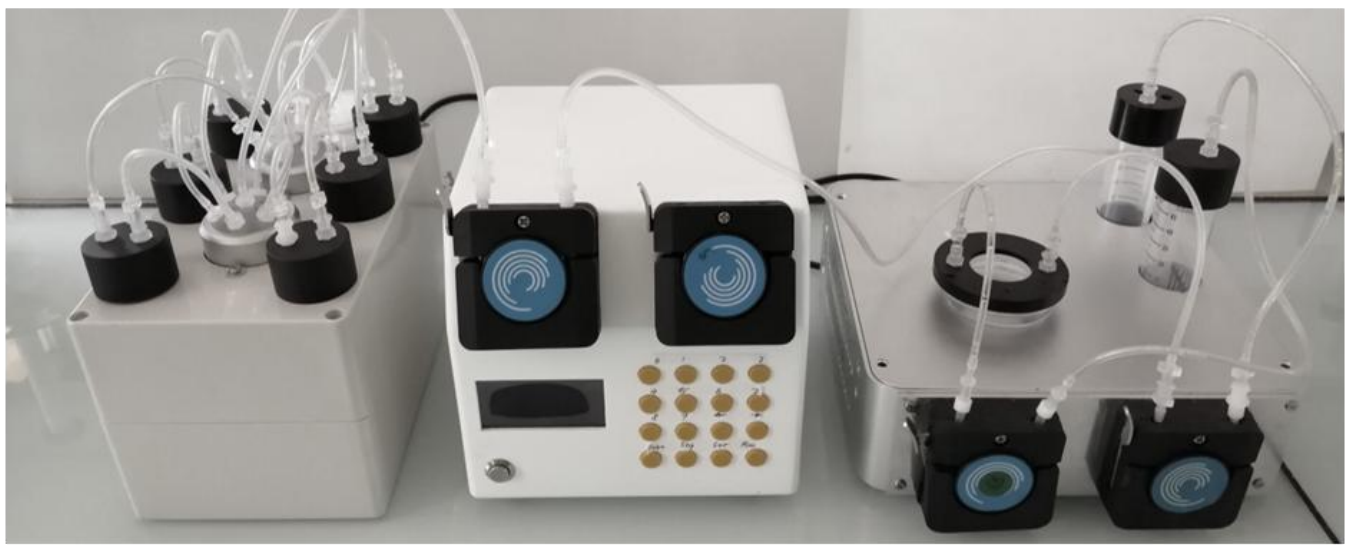

Storage unit

Control unit

Cell culture unit

(b)

(c)

(d)
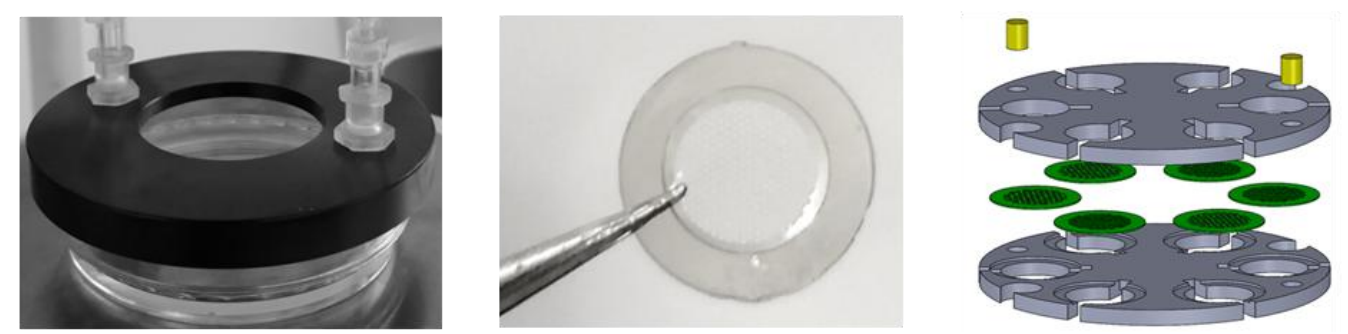
Fig.3

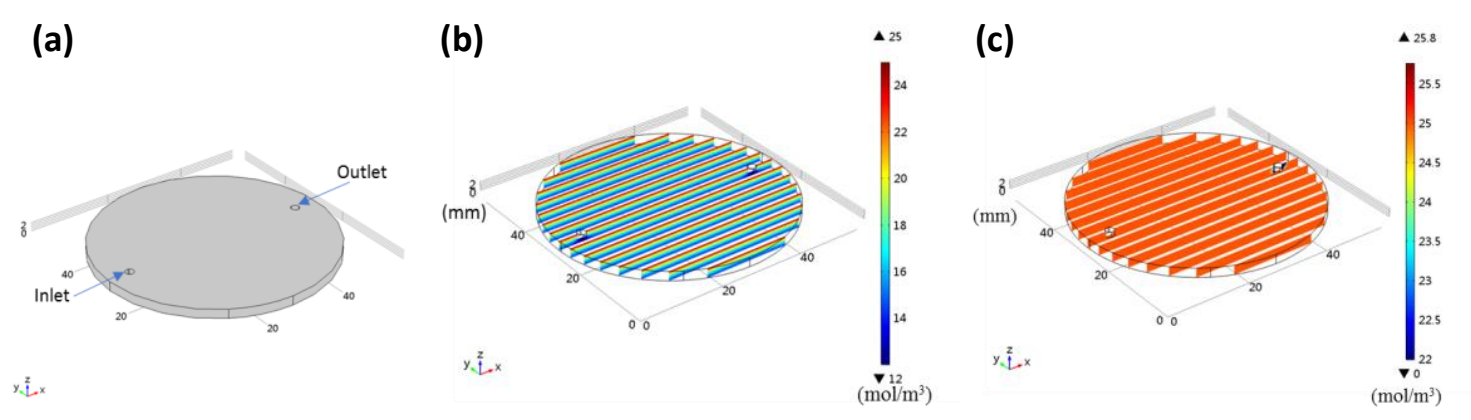

Fig. 4

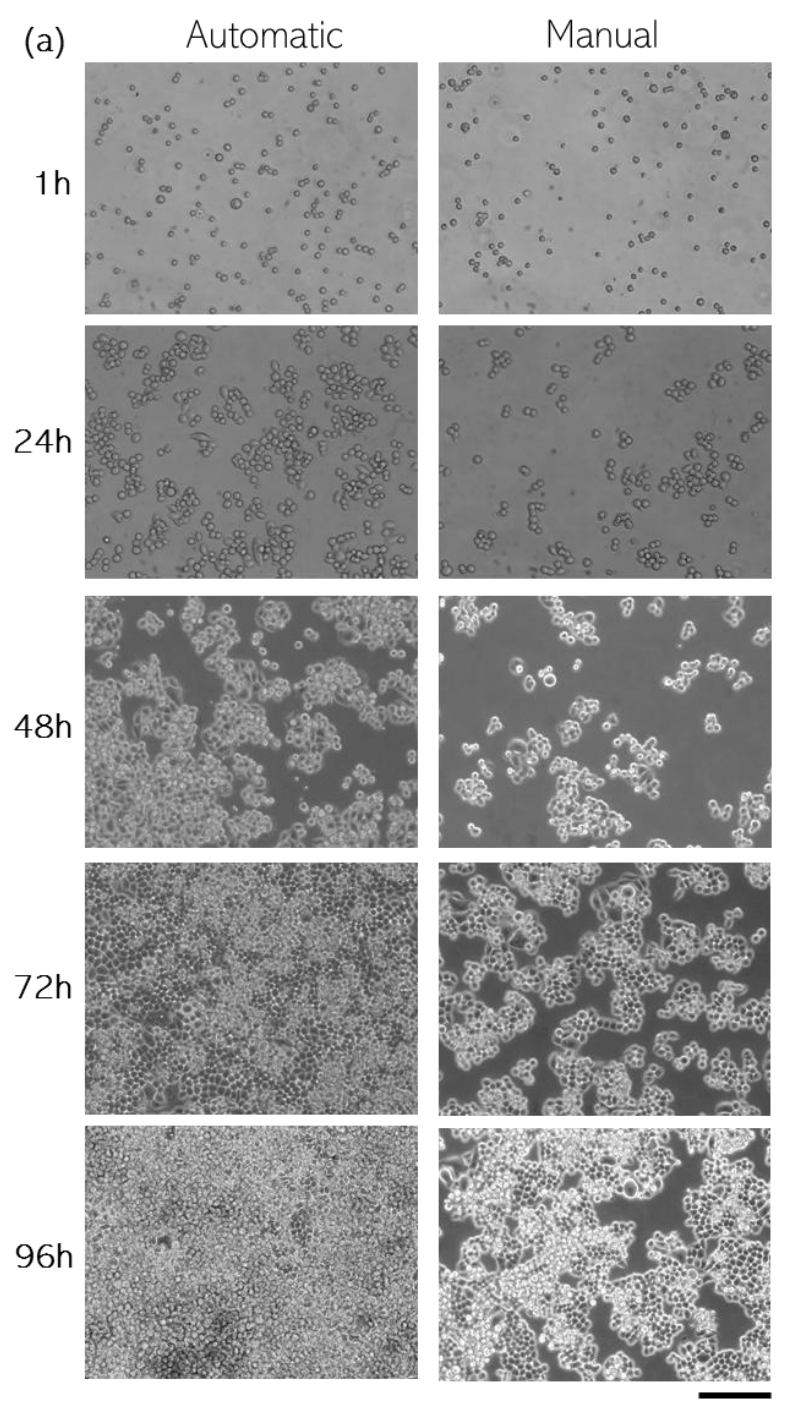

(b)
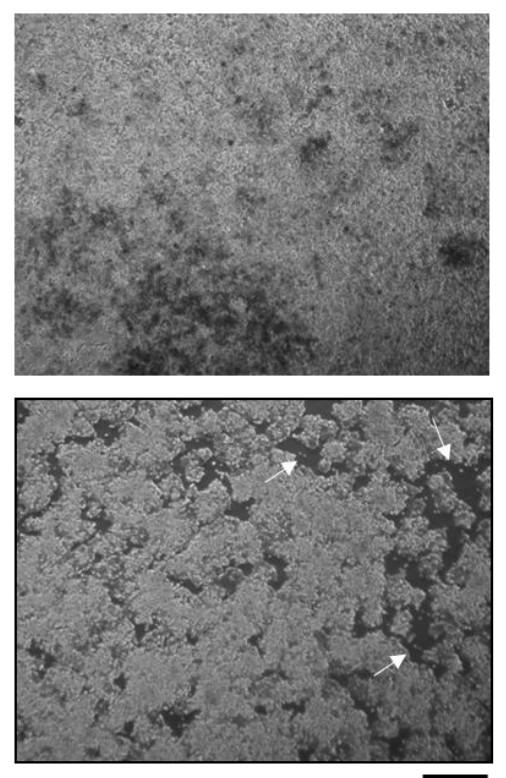

(c)

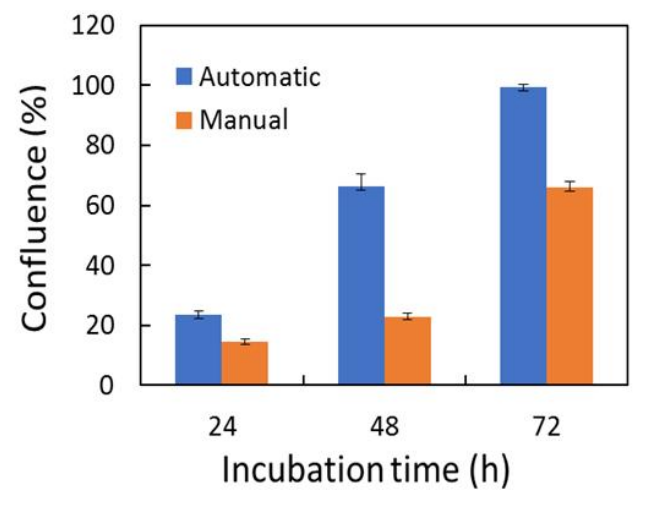


Fig. 5

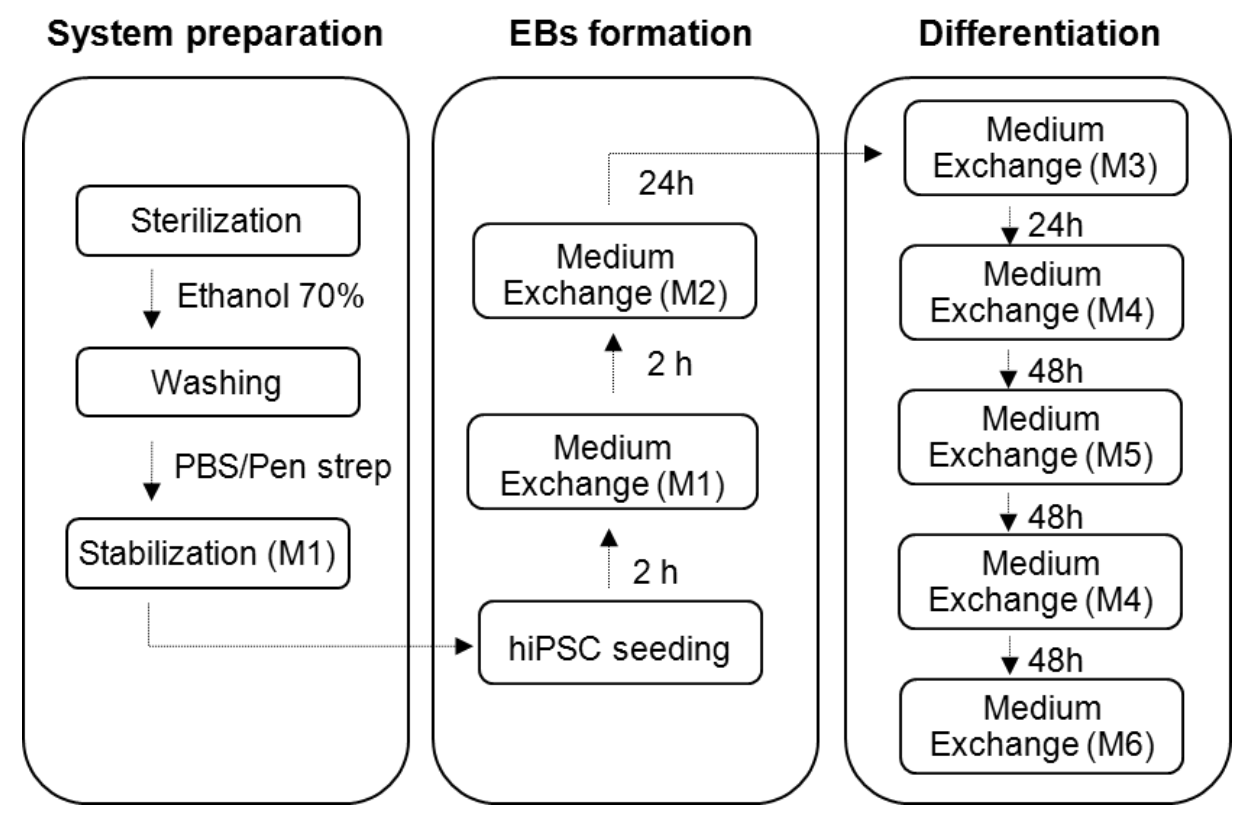

Figure 6

(a)

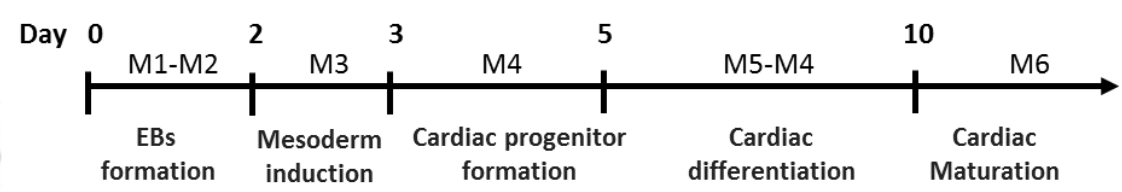

(b)

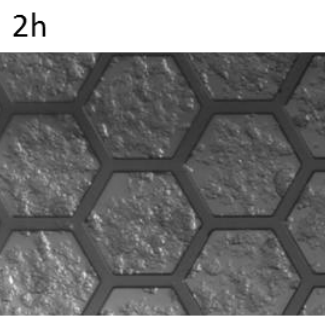

(c)

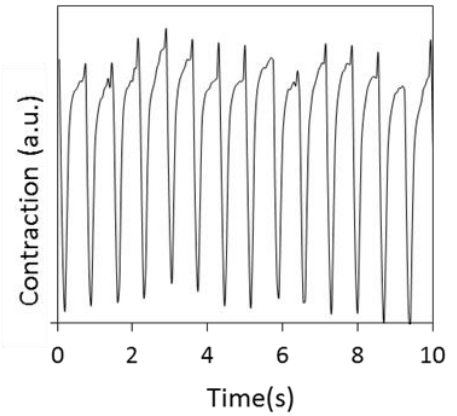

D1

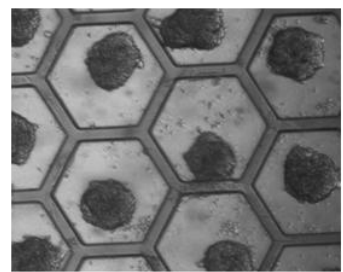

(d)

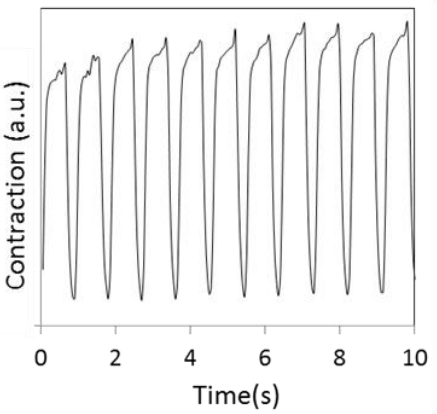

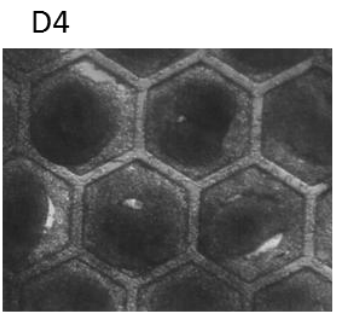

D20

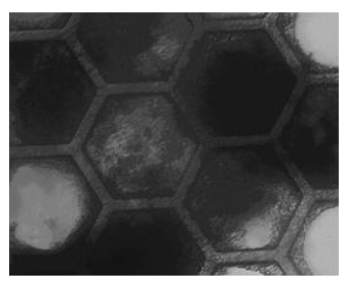

- $200 \mu \mathrm{m}$

(e)

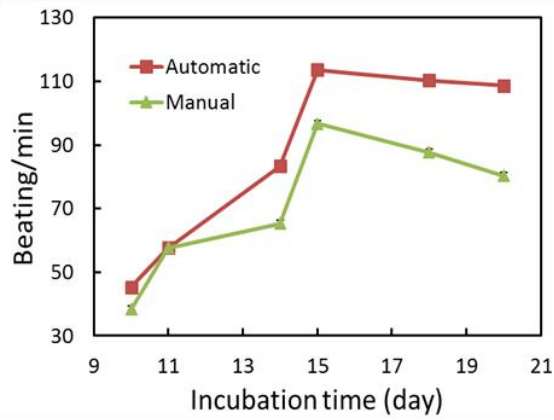

